\title{
PULVERIZAÇÃO ELETROSTÁTICA COM BICOS HIDRÁULICOS
}

ADEMIR CHAIM*

\begin{abstract}
Sistema de indução por eletrificação direta para bicos hidráulicos foi testado em condições de laboratório para obtenção de parâmetros de voltagens, correntes de eletrificação e distâncias de pulverização mais adequados para o desenvolvimento de pulverizador costal eletrostático. Foram testados os bicos TXVS2, TXVK-3 e TXVK-4 em associação com tensões de $-40 \mathrm{kV},-32 \mathrm{kV}$ e $-25 \mathrm{kV}$. Resistor de $1 \mathrm{G} \Omega$ limitou a potência teórica máxima da fonte de alta tensão em 1,6 watts para $40 \mathrm{kV}$. Foram utilizados 4 tubos metálicos aterrados, radialmente dispostos em relação ao bico, em distâncias de $25 \mathrm{~cm}, 30 \mathrm{~cm}$, $35 \mathrm{~cm}$ e $40 \mathrm{~cm}$ para coletar as gotas eletrificadas. $O$ bico TXVK-3, alimentado com $-40 \mathrm{kV}$, proporcionou recuperação média de $678 \mathrm{~mL}$ para cada litro pulverizado numa distância de $25 \mathrm{~cm}$ dos tubos coletores. A recuperação diminuiu com a redução da tensão e com o aumento da distância dos alvos da pulverização. Todos os bicos testados recuperaram mais de $30 \%$ dos volumes aplicados, com tensões entre 32 e $40 \mathrm{kV}$ e distância mínima de $30 \mathrm{~cm}$ dos alvos.
\end{abstract}

PALAVRAS-CHAVE: AGROTÓXICO; BICOS HIDRÁULICOS; GOTAS DE PULVERIZAÇÃO; PULVERIZADOR COSTAL ELETROSTÁTICO.

* Engenheiro Agrônomo, M. Sc. em Tecnologia de Aplicação de Agrotóxicos, Embrapa Meio Ambiente, Jaguariuna, SP (e-mail: aldemir@cnpma.embrapa.br). 


\section{INTRODUÇÃO}

As deposições de agrotóxicos são ineficientes em quase todas formas de aplicação e tipo de cultura. Em pulverização aérea, por exemplo, foram verificadas perdas em torno de $50 \%$ do volume de calda aplicado (PESSOA e CHAIM, 1999). Em cultura de porte rasteiro, como tomate e feijão, as perdas variam entre $48 \%$ a $88 \%$ (CHAIM et al., 1999a). Além disso, a deposição nas culturas de porte rasteiro concentra-se na região do ponteiro das plantas (CHAIM, VALARINI e PIO, 2000; SCRAMIN et al., 2002). Em culturas de porte arbustivo, como tomateiro estaqueado, foram verificadas perdas entre 59\% a 76\% (CHAIM et al.,1999b). Em videira, as perdas variaram entre $18 \%$ a $39 \%$ dependendo do tipo de equipamento e bico de pulverização (CHAIM, PESSOA e FERRACINI, 2004). Em culturas de porte arbóreo, como a macieira, verificaram-se perdas entre $32 \%$ a $40 \%$ de acordo com a variedade da fruteira (CHAIM et al., 2003).

$\mathrm{Na}$ tentativa de melhorar a eficiência da aplicação dos agrotóxicos desenvolveram-se pesquisas para aumentar a deposição nos alvos e reduzir a contaminação ambiental. As pesquisas sobre o uso de gotas com cargas eletrostáticas evidenciaram grande evolução, principalmente, depois do sucesso do pulverizador manual Electrodyn (COFFE, 1981) utilizado no nordeste brasileiro contra pragas do algodão, entre 1980 e 1990. Pulverizador manual eletrohidrodinâmico foi desenvolvido no Brasil (CHAIM, 1984), entre 1982 e 1984, para aplicação de volumes de calda entre 1 a 2 litros por hectare. Sua utilização alcançou sucesso no controle de trips em amendoim, empregando apenas $50 \%$ da dose recomendada de deltametrina. No Brasil, também foram desenvolvidos bicos pneumáticos eletrostáticos para equipamentos tratorizados (CHAIM, 1998; CHAIM, LARANJEIRO e CAPALBO, 1999) e bocal eletrostático adaptável para pulverizadores motorizados costais (CHAIM, PESSOA e FERRACINI, 2002).

Experimentos de campo comprovaram redução de aproximadamente 5 vezes a dose de ingrediente ativo (COFFE, 1981; ARAUJO et al., 2002) em relação a pulverização convencional. Essa tecnologia ainda não adquiriu consistência, apesar de algumas pesquisas constatarem aumento expressivo na deposição proporcionada pela pulverização eletrostática (CHAIM, PESSOA e FERRACINI, 2002).

O sistema eletrohidrodinâmico (COFFE, 1981; CHAIM, 1984) necessita formulações especiais, baseadas em óleo, com custos de registros elevados. Além disso, nem todos os ingredientes ativos de agrotóxicos apresentam características adequadas para serem uniformemente dissolvidos em óleo. Isso limita o emprego dessa tecnologia para pragas específicas de algumas culturas.

O emprego de caldas aquosas, com condutividade elétrica razoavelmente elevada, exige processo de indução com carga indireta (CHAIM, 1998; CHAIM, LARANJEIRO e CAPALBO, 1998; CHAIM, PESSOA e FERRACINI, 2002) em que o líquido deve ser mantido aterrado, ou seja, em zero volt. Para ocorrer a eletrificação das gotas no processo de indução com carga indireta é necessária a manutenção do eletrodo de indução de alta tensão posicionado próximo a zona de formação das gotas. O eletrodo possibilita a formação de intenso campo eletrostático, induzindo o aparecimento de íons na superfície do líquido que emerge do bico. Conforme as gotas são formadas levam consigo os íons acumulados e adquirem carga. Entretanto, os íons presentes na superfície das gotas apresentam polaridade oposta ao eletrodo de alta tensão. Como o campo eletrostático é elevado, as gotas são fortemente atraídas para o eletrodo que ao ser molhado degrada todo o processo. O problema pode ser solucionado utilizando-se ar em alta velocidade para manter 0 eletrodo de alta tensão seco (CHAIM, 1998; CHAIM, LARANJEIRO e CAPALBO, 1999; CHAIM, PESSOA e FERRACINI, 2002). Como o bombeamento de ar consome muita energia, essa tecnologia não é adequada para desenvolvimento de equipamentos hidráulicos em que o líquido é bombeado manualmente.

A eficiência da pulverização eletrostática está inversamente relacionada ao tamanho das gotas e diretamente relacionada com a intensidade de carga (CHAIM, 1998; CHAIM, LARANJEIRO e CAPALBO, 1999; CHAIM, PESSOA e FERRACINI, 2002). Algumas empresas colocam no mercado pulverizadores eletrostáticos que não atendem as necessidades de intensidade de carga e tamanho 
adequado de gotas para incrementar expressivamente a deposição. Esse tem sido, provavelmente, o principal empecilho para a adoção mais rápida da tecnologia eletrostática.

O processo eletrohidrodinâmico produz gotas com elevadíssima intensidade de carga, mediante ação exclusiva de forças criadas por intenso campo eletrostático (CHAIM, 1984). Por esse motivo, utiliza-se o sistema de carga por indução com eletrificação direta em que o eletrodo aterrado promove o campo eletrostático necessário para romper o líquido em gotas. A utilização do sistema de carga por indução com eletrificação direta, em bicos hidráulicos, exigiria a eliminação do eletrodo aterrado para evitar a atração das gotas eletrificadas. Alternativa para obter campo eletrostático intenso, nesse caso, seria aumentar a tensão de eletrificação ao ponto em que a indução fosse estabelecida entre o bico eletrificado e as plantas aterradas. Todo o circuito hidráulico do pulverizador ficaria submetido a alta tensão pelo fato da calda aquosa ser eletricamente condutora, criando riscos de choques ao aplicador. Como a carga das gotas é proporcional a intensidade do campo eletrostático formado (CHAIM, 1998; CHAIM, LARANJEIRO e CAPALBO, 1999; CHAIM, PESSOA e FERRACINI, 2002) seria necessário estabelecer a tensão e a distância mais adequadas para maximização da deposição.

O objetivo deste trabalho foi testar sistema de indução por eletrificação direta em bicos hidráulicos, em condições de laboratório, visando a obtenção dos parâmetros de voltagens, correntes de eletrificação e distâncias de pulverização mais adequados para futuro desenvolvimento de pulverizador hidráulico eletrostático costal manual.

\section{MATERIAL E MÉTODOS}

Os testes foram realizados nos galpão do Laboratório de Tecnologia de Aplicação de Agrotóxicos da Embrapa Meio Ambiente. Para eletrificação do jato de gotas, uma fonte especial de alta tensão foi desenvolvida pela Empresa Tecnovance Equipamentos para Pintura (Jundiaí - SP). A fonte alimentada com $6 \mathrm{~V} / 500 \mathrm{~mA}$ (potência de 3,0 watts) apresentava voltagens pré-calibradas em - 40 kV, -32 kV, -25kV e 0 V com aproximadamente $1 \%$ de precisão.

Os testes consistiram na coleta de calda pulverizada por meio de quatro tubos metálicos de aproximadamente $80 \mathrm{~cm}$ de altura e $10 \mathrm{~cm}$ de diâmetro (Figura 1). Os tubos metálicos e a fonte foram aterrados no solo e a água coletada em placas de Petri. O bico ficou posicionado na região central da distribuição dos tubos há aproximadamente $60 \mathrm{~cm}$ de altura.

Desenvolveu-se pulverizador hidráulico especial, utilizando garrafas PET de refrigerantes com capacidade de 2 litros como tanque de água. O líquido foi pressurizado continuamente com ar comprimido, sendo o controle de pressão ajustado por válvula que regulava o escape do ar. Montouse o sistema sobre uma base isolada de acrílico com $50 \mathrm{~cm}$ por $50 \mathrm{~cm}$ e espessura de 0,8 cm. Reforço na capacidade de isolação das mangueiras do circuito hidráulico foi obtido com tubo de pvc. Assim, a mangueira que ligava o tanque ao bico foi embutida em tubo de pvc (com $150 \mathrm{~cm}$ de comprimento por $2,4 \mathrm{~cm}$ de diâmetro) fixado ao meio de uma das laterais da base de acrílico. Efetuou-se a eletrificação direta na calda de pulverização, mediante dispositivo metálico pontiagudo embutido no suporte do bico.

Foram utilizados os bicos tipo cone, TXVS-2 em $120 \mathrm{~mL} / \mathrm{min}$, TXVK-3 em $185 \mathrm{~mL} / \mathrm{min}$ e o TXVK-4 em $250 \mathrm{~mL} / \mathrm{min}$ de vazão em pressão calibrada em $2,5 \mathrm{~kg} / \mathrm{cm}^{2}$. Em cada teste, efetuou-se pulverização exata de 1 litro de água com controle preciso estabelecido por cronômetro. Os tempos em que cada bico pulverizava um litro foram: 500 segundos para o bico TXVS-2, 324 segundos para o bico TXVK-3 e 240 segundos para o bico TXVK-4. Testes prévios indicaram que a deposição nos tubos de alumínio com os bicos operando sem carga era praticamente nula em qualquer distância de pulverização de até o mínimo de $20 \mathrm{~cm}$. Assim, o controle do tempo de pulverização foi realizado ligando-se ou desligando-se a fonte de alta tensão. A fonte era desligada no final do tempo de pulverização de um litro, cessando completamente a atração e conseqüentemente a deposição nos tubos coletores, apesar do bico continuar pulverizando normalmente. 


\section{FIGURA 1 - DISTRIBUIÇÃO DE TUBOS COLETORES DE LÍQUIDO EM RELAÇÃO AO BICO PARA TESTE DE EFICIÊNCIA DE DEPOSIÇÃO DE PULVERIZAÇÃO ELETROSTÁTICA}

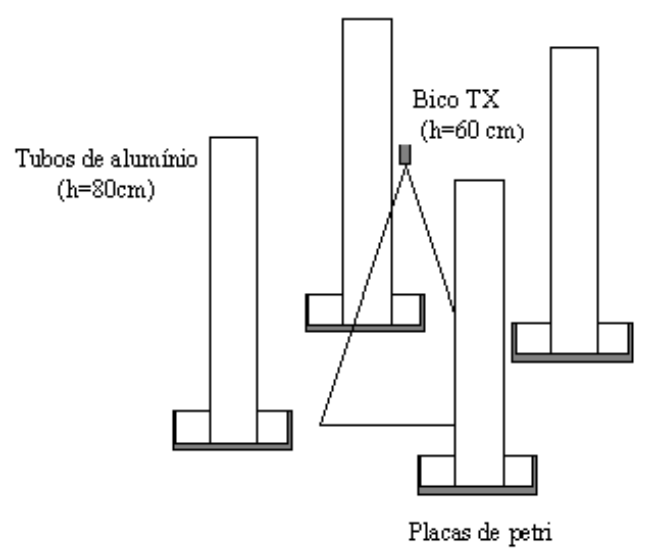

Os testes foram divididos em dois experimentos básicos, sendo que o primeiro consistiu na verificação da influência da redução da corrente de eletrificação do jato de gotas. Nesse caso, utilizou-se o bico TXVS-2 a $30 \mathrm{~cm}$ dos coletores. Combinaram-se as tensões de $-40 \mathrm{kV},-32 \mathrm{kV}$ e - $25 \mathrm{kV}$ com os resistores de $1 \mathrm{G} \Omega, 2,0 \mathrm{G} \Omega$ e 4,0 G $\Omega$ (1\% de precisão), fornecidos pela Tecnoavance. Foram realizadas 5 repetições de coletas de calda pulverizada de cada tratamento. Devido à elevadíssima tensão empregada na eletrificação direta da calda de pulverização testou-se o método de CHAIM (1998) para medir a carga eletrostática do jato de gotas, utilizando microamperímetro analógico Engro (modelo 455L) e coletando gotas a $10 \mathrm{~cm}$ de distância do bico TXVS-2 (120 mL/ $\mathrm{min}$ ). Esse teste foi realizado empregando-se a fonte calibrada em $-40 \mathrm{kV}$, os resistores em série de $1 \mathrm{G} \Omega, 2,0 \mathrm{G} \Omega$ e sem resistor. O segundo experimento consistiu na verificação da influência dos bicos TXVS-2, TXVK-3 e TXVK-4 na deposição. Combinaram-se com as distâncias de pulverização de $25 \mathrm{~cm}, 30 \mathrm{~cm}, 35 \mathrm{~cm}$ e $40 \mathrm{~cm}$ e as voltagens de $-40 \mathrm{kV},-32 \mathrm{kV}$ e $-25 \mathrm{kV}$ com a melhor associação do resistor apontado no experimento anterior. Nesse tipo de delineamento em parcela subdividida (BANZATO e KRONCA, 1995), os bicos foram considerados como tratamentos principais e tensões e distâncias de pulverização como tratamentos secundários. Para exemplificar um dos blocos incluiu os seguintes tratamentos: TXVK-3/-40 kV $+40 \mathrm{~cm},+35 \mathrm{~cm},+30 \mathrm{~cm},+25 \mathrm{~cm} ;$ TXVK-3/-32 kV $+40 \mathrm{~cm}$, $+35 \mathrm{~cm},+30 \mathrm{~cm},+25 \mathrm{~cm}$; TXVK-3/-25 kV $+40 \mathrm{~cm},+35 \mathrm{~cm},+30 \mathrm{~cm},+25 \mathrm{~cm}$. Outros blocos seguiram o mesmo raciocínio, mas com tratamentos executados em seqüência aleatória. Foram realizadas 5 repetições alternadas entre os blocos dos diferentes tratamentos principais.

Os testes foram realizados em períodos matinais, com condições de temperatura variando entre os limites de 23 e $27^{\circ} \mathrm{C}$ e umidade relativa entre e $56 \%$ e $74 \%$, monitorados com termohigrômetro digital Sper Scientific 800016.

\section{RESULTADOS E DISCUSSÃO}

A fonte de alta tensão utilizada nos experimentos apresentou potência teórica de 3 Watts. Assumindo eficiência de conversão de 100\%, a corrente teórica máxima de saída seria de 75 microampères para $40 \mathrm{kV}$. Pela lei de $\mathrm{Ohm}, \mathrm{I}=\mathrm{V} / \mathrm{R}$, o I é a corrente (ampère), $\mathrm{V}$ a tensão (volt) e $\mathrm{R}$ a resistência (ohm) (BRAGA, 1985). Desta forma, ao associar-se resistor de $1 \mathrm{G} \Omega$ em série com a fonte em $40 \mathrm{kV}$, a corrente de saída na alta tensão seria no máximo de 40 microampères. Para resistores de $2 \mathrm{G} \Omega$ e $4 \mathrm{G} \Omega$ a corrente seria respectivamente de 20 e 10 microampères para tensão de $40 \mathrm{kV}$. Com esses resistores associados em série com a fonte, testes prévios apontaram que qualquer pessoa devidamente aterrada ou em contato com o solo não sentiria nenhuma sensação 
de "choque" ao tocar o terminal de AT. Além desse aspecto benéfico, o resistor associado com a fonte aumentaria a segurança para o aplicador. No caso de alguma descarga acidental, a potência final seria inferior a 1,6 watts. O efeito da redução de corrente da alta tensão do sistema de carga por indução com eletrificação direta em bico hidráulico pode ser verificado na Tabela 1.

\section{TABELA 1 - VOLUME DE LÍQUIDO COLETADO (mL/Litro PULVERIZADO) EM FUNÇÃO DE DIFERENTES VOLTAGENS APLICADAS AO BICO DE PULVERIZAÇÃO TXVS-2 (120 mL/min) E DIFERENTES RESISTORES PARA REDUÇÃO DE CORRENTE}

\begin{tabular}{ccccc}
\hline Voltagem $(\mathrm{kV})$ & Fonte normal & Fonte $+1 \mathrm{G} \Omega$ & Fonte $+2 \mathrm{G} \Omega$ & $\mathrm{F}$ onte $+4 \mathrm{G} \Omega$ \\
\hline 0 & 0 & 0 & 0 & 0 \\
-25 & 270 & 268 & 178 & 136 \\
-32 & 364 & 366 & 300 & 240 \\
-40 & 420 & 392 & 364 & 310 \\
\hline
\end{tabular}

Considerando os requisitos de segurança, o consumo de energia e a deposição, a fonte poderia trabalhar entre $-32 \mathrm{kV}$ e $-40 \mathrm{kV}$ com resistor de $1 \mathrm{G} \Omega$. Entre essas duas tensões a diferença de deposição seria de apenas $7 \%$. Algumas observações durante a realização dos testes revelaram que a associação da fonte com resistor de $4 \mathrm{G} \Omega$ deveria ser descartada. Com a baixíssima corrente liberada, o sistema de carga fica instável em condições de umidade relativa elevada, provavelmente, devido as fugas facilitadas pela redução da capacidade de isolamento do ar. Sob condição de UR acima de 76\%, a aproximação de objetos aterrados do tanque (mesmo em distâncias superiores a $40 \mathrm{~cm}$ ), de componentes do circuito hidráulico, ou do bico afetaram negativamente a deposição.

No teste de medição da carga eletrostática do jato de gotas verificou-se que a corrente do jato ultrapassava $-30 \mu \mathrm{A}$, com a fonte $(-40 \mathrm{kV})$ sem resistor. Utilizando resistores de $2 \mathrm{G} \Omega$ e $1 \mathrm{G} \Omega$ em série com a fonte, a corrente ficou respectivamente em torno de $-8,5 \mu \mathrm{A}( \pm-0,5 \mu \mathrm{A})$ e $-13,5 \mu \mathrm{A}( \pm-0,5 \mu \mathrm{A})$. Escolheu-se a distância de $10 \mathrm{~cm}$ para permitir a leitura de carga apenas de gotas completamente formadas, sem elos de ligação com o bico eletrificado. Esse método adaptado de CHAIM (1998) não se mostrou adequado ao processo de eletrificação direta, pois a corrente seria alterada por influência do elevadíssimo campo eletrostático se a medição fosse efetuada numa distância diferente de $10 \mathrm{~cm}$ em relação ao bico. Contudo, aceitou-se que o resistor de $1,0 \mathrm{G} \Omega$ fosse o mais adequado, pois a $10 \mathrm{~cm}$ de distância a carga liberada pelo jato de gotas pelo bico TXVS-2 (120 mL/min) ficaria entre -6 a -7 milicoulomb/Litro. O resultado -6 a $-7 \mathrm{mC} / \mathrm{L}$ foi obtido pela divisão das corrente -0,013 mA a -0,014 mA pela vazão de 0,002 L/segundo (CHAIM, 1998).

A Tabela 2 ilustra a deposição em termos percentuais em relação a fonte normal, considerando o efeito dos resistores na redução da intensidade de corrente no jato de gotas. Essa forma de avaliação reforça a garantia de que o resistor de $1 \mathrm{G} \Omega$ é o mais adequado para ser associado à fonte de AT para eletrificação das gotas.

\section{TABELA 2 - PERCENTUAL DE DEPOSIÇÃO DE LÍQUIDO CONSIDERANDO DIFERENTES RESISTORES LIMITADORES DE CORRENTE DE ELETRIFICAÇÃO DO JATO DE GOTAS (BICO TXVS-2, $120 \mathrm{~mL} / \mathrm{min}$ )}

\begin{tabular}{ccccc}
\hline Voltagem $(\mathrm{kV})$ & Fonte normal & $\mathrm{F}+1 \mathrm{G} \Omega$ & $\mathrm{F}+2 \mathrm{G} \Omega$ & $\mathrm{F}+4 \mathrm{G} \Omega$ \\
\hline-25 & 100 & 99 & 66 & 50 \\
-32 & 100 & 101 & 82 & 66 \\
-40 & 100 & 95 & 88 & 74 \\
\hline
\end{tabular}


Os resultados da comparação dos bicos, voltagens e distâncias de coleta de pulverização são apresentados na Tabela 3. Notou-se que o volume coletado de líquido diminuiu com a redução da voltagem e aumento da distância. O bico que demonstrou os melhores índices de deposição foi o TXVK-3, provavelmente porque suas gotas apresentaram o melhor tamanho para serem atraídas sem sofrer efeitos de evaporação. Apesar do bico TXVS-2 fornecer o melhor tamanho de gotas para a eletrodeposição nas folhas das plantas, a evaporação das pequenas gotas pode ter sido intensa e reduziu o volume coletado. O bico TXVK-4, teoricamente, produziu porcentagem de gotas razoavelmente grandes, sendo a força eletrostática insuficiente para vencer a gravidade. A carga eletrostática não evitou que parte expressiva de gotas grandes se projetasse diretamente no solo, não só pela ação da força da gravidade, mas também pela velocidade de arremesso descendente e vertical do jato.

\section{TABELA 3 - VOLUME MÉDIO DE LÍQUIDO COLETADO (mL/LITRO PULVERIZADO) EM FUNÇÃO DE DIFERENTES VOLTAGENS APLICADAS, DIFERENTES BICOS E DISTÂNCIAS DE PULVERIZAÇÃO, UTILIZANDO FONTE DE AT EM SÉRIE COM RESISTOR DE 1 G $\Omega$}

\begin{tabular}{lcccc}
\hline Bicos tipo cone & $\begin{array}{c}\text { Distâncias dos coletores } \\
\text { em relação ao bico }(\mathrm{cm})\end{array}$ & -40 & -32 & -25 \\
\hline \multirow{3}{*}{ TXVS-2 } & 25 & 560 & 508 & 430 \\
& 30 & 390 & 320 & 250 \\
& 35 & 283 & 228 & 153 \\
& 40 & 200 & 150 & 100 \\
TXVK -3 & & & \\
& 25 & 678 & 600 & 503 \\
& 30 & 500 & 443 & 340 \\
& 35 & 380 & 293 & 210 \\
TXVK -4 & 270 & 180 & 125 \\
& 40 & & & \\
& 25 & 588 & 508 & 428 \\
& 30 & 448 & 380 & 320 \\
& 35 & 340 & 270 & 205 \\
& 40 & 230 & 165 & 100 \\
\hline
\end{tabular}

A Tabela 3 fornece boa estimativa da potencialidade da eletrificação das gotas. Apenas 4 tubos coletores com $10 \mathrm{~cm}$ de diâmetro, eqüidistantes, conseguiram recuperar aproximadamente $70 \%$ do volume de calda pulverizado com o bico TKVK-3 a $25 \mathrm{~cm}$ de distância. Se houvessem mais tubos coletores, a coleta poderia atingir facilmente volumes próximos a $100 \%$ demonstrando a fortíssima atração existente entre as gotas eletrificadas e os alvos aterrados. O volume recuperado (70\%) deve ser comparado com o volume aplicado, mas não com a pulverização de bico sem carga em que a recuperação seria $0 \%$.

Dependendo da arquitetura das plantas, o bico de pulverização poderia ser transportado nas entrelinhas da cultura facilitando a penetração das gotas no dossel para atingir a face inferior das folhas. Nessa situação de transporte do bico, as perdas para o solo seriam reduzidas.

Os desdobramentos da Análise de Variância (BANZATO e KRONCA, 1995) para verificar os efeitos entre os tratamentos são apresentados pelos resultados de aplicação de teste $F$ (Tabela 4). Notou-se que as voltagens apresentaram os maiores valores de $F$ quando se analisa o seu efeito nas distâncias de amostragem. Esse efeito é justificado pelo enunciado da Lei de Coulomb (COFFE, 1981) que afirma que a força de atração eletrostática é diretamente proporcional ao produto das cargas e inversamente proporcional ao quadrado da distância. A carga é influenciada pela tensão aplicada, vazão do bico (tamanho das gotas) e principalmente pelo campo eletrostático, 
que pode ser expressa de maneira simplificada (COFFE, 1981) como: $E=V / r$, em que $E=0$ campo eletrostático $(\mathrm{V} / \mathrm{m}), \mathrm{V}=\mathrm{a}$ voltagem (volt) e $\mathrm{r}=$ a distância entre o jato de gotas e o alvo de indução (m). Os resultados apresentados nas Tabelas 3 e 4 comprovam que a intensidade do campo eletrostático é fundamental para o sistema de indução por carga direta, pois a distância foi o parâmetro de maior influência para a deposição.

A identificação (Tabelas 3 e 4) de que a variação da voltagem exerce efeito menos expressivo na deposição que a distância torna-se valiosa para o desenvolvimento de pulverizador hidráulico eletrostático costal, que seria alimentado por pilhas ou bateria recarregável. A fonte de alta tensão poderia enquadrar-se na amplitude de 30 a 40 kV com recuperação considerável de deposição, aproveitando o máximo da carga das pilhas ou bateria. Entretanto, o bico do pulverizador deveria ser mantido na distância máxima de $30 \mathrm{~cm}$ das plantas para se obter recuperação mínima de $30 \%$ do volume pulverizado.

\section{TABELA 4 - VALORES DE $F^{(1)}$ CALCULADOS PARA DESDOBRAMENTOS DA ANÁLISE DE VARIÂNCIA DOS TRATAMENTOS}

\begin{tabular}{|c|c|c|c|}
\hline \multirow{2}{*}{$\begin{array}{l}\text { Causas de } \\
\text { Variação }\end{array}$} & \multicolumn{3}{|c|}{ Efeito das causas de variação em cada parâmetro } \\
\hline & Bico & Distância & Voltagem \\
\hline$-40 \mathrm{kV}$ & $350,7^{\star \star}$ & $4435,7^{* *}$ & \\
\hline$-32 \mathrm{Kv}$ & $329,6^{\star *}$ & 2194,6 ** & \\
\hline$-25 \mathrm{kV}$ & $168,8^{\star \star}$ & 1866,9 ** & \\
\hline TXVS-2 & & 2971,9 ** & $597,6^{* *}$ \\
\hline TXVK-3 & & 2712,9 ** & $1161,6^{* *}$ \\
\hline TXVK-4 & & $242,7^{* *}$ & $101,7^{\star \star}$ \\
\hline $25 \mathrm{~cm}$ & $348,7^{* *}$ & & $934,9^{* *}$ \\
\hline $30 \mathrm{~cm}$ & 246,9 ** & & $776,4^{\star *}$ \\
\hline $35 \mathrm{~cm}$ & $201,0 * \star$ & & $852,5^{\star \star}$ \\
\hline $40 \mathrm{~cm}$ & $206,1^{\star *}$ & & $705,2^{\star *}$ \\
\hline
\end{tabular}

(1) Para $F^{\star \star}$ efeito significativo ao nível de $1 \%$ de probabilidade.

Os resultados dos testes comprovam a possibilidade de se adaptar, ou construir pulverizador costal com bicos hidráulicos convencionais para pulverização eletrostática por indução com eletrificação direta. O mercado já dispõe de equipamentos totalmente construídos com materiais plásticos e boas características de isolação para altas tensões. Entretanto, ainda seriam necessárias algumas adaptações no circuito hidráulico e formato do tanque para reduzir o risco de fuga de alta tensão. Apesar de exigir tensão elevada, entre 30 e $40 \mathrm{kV}$, a potência final da fonte de alta tensão estaria entre 1,2 a 1,6 watts. O aplicador não sentiria choques elétricos, desde que devidamente aterrado com calçado com sola condutora, mas a isolação do equipamento seria necessária para evitar que eventuais fugas de alta tensão reduzissem a energia disponibilizada para a eletrificação do jato de gotas.

\section{CONCLUSÃO}

O sistema de carga por indução com eletrificação direta, aplicado em bicos hidráulicos convencionais, proporcionou elevados índices de recuperação do volume pulverizado em alvos artificiais. A melhor faixa de tensão necessária para a eletrificação do jato de gotas situou-se entre 30 e $40 \mathrm{kV}$, mas com potência final baixa (entre 1,2 a 1,6 watts). A distância que o bico deve ser posicionado em relação ao alvo foi o efeito mais significativo no sistema de carga por indução com eletrificação direta. Devido a baixa potência necessária, esse sistema pode ser adaptado em pulverizadores costais convencionais, desde que sejam tomados os cuidados necessários para a eliminação de fuga de alta tensão. 


\section{ABSTRACT \\ ELECTROSTATIC SPRAYING WITH HIDRAULIC NOZZLES}

The purpose of this research was to test, in laboratory conditions, an induction system by direct electrification of hydraulic nozzles, which establishes the most adequate parameters of voltages, electrification currents and spraying distances, for the development of knapsack electrostatic sprayer. The hydraulic hollow cone nozzles TXVS-2, TXVK-3 and TXVK-4 were tested in association with high voltages of $-40 \mathrm{kV},-32 \mathrm{kV}$ and $-25 \mathrm{kV}$. A $1 \mathrm{G} \Omega$ resistor was used to limit the maximum theoretical power of the high-tension source, in 1,6 watts for $40 \mathrm{kV}$. Four grounded metallic tubes in radial display were used with distances of $25 \mathrm{~cm}, 30 \mathrm{~cm}, 35 \mathrm{~cm}$ and $40 \mathrm{~cm}$ from the nozzle, to collect the electrified droplets. The hollow cone nozzle TXVK-3 powered with $-40 \mathrm{kV}$ provided an average recovery of $678 \mathrm{~mL}$ for each sprayed litre, at $25 \mathrm{~cm}$ from the collector's tubes. The recovery decreased with the reduction of the voltage and with the increase of the spraying distance to the targets. All the nozzles tested recovered more than $30 \%$ of the applied volumes, with voltages between $-32 \mathrm{kV}$ and $-40 \mathrm{kV}$ and minimum distance of $30 \mathrm{~cm}$ from the targets.

KEY - WORDS: PESTICIDE; HIDRAULIC NOZZLES; SPRAYING DROPLETS; KNAPSACK ELECTROSTATIC SPRAYER.

\section{REFERÊNCIAS}

1 ARAUJO, L. H. A.; SANTOS, R. F.; SOUSA, S. L.; QUEIROGA, V. P.; LIMA, N. J. Avaliação da mistura endosulfan com óleos vegetais para o controle do bicudo do algodoeiro, Anthonomus Grandis Boheman,1843 (coleoptera:curculionidae). Campina Grande: Embrapa Algodão, 2002. 6 p. (Comunicado Técnico, 151).

2 BANZATO D.A.; KRONCA, S.N. Experimentação agrícola. 3.ed. Jaboticabal: FUNEP, 1995. 247 p.

3 BRAGA, N.C. Circuitos \& informações. São Paulo: Editora Saber, 1985. 158 p. (Coleção Saber Eletrônica, v.1)

4 CHAIM, A. Desenvolvimento de um pulverizador eletrohidrodinâmico: avaliação do seu comportamento na produção de gotas e controle de trips (Enneothrips flavens Moulton, 1951), em amendoim (Arachis hypogaea L.). Jaboticabal, 1984. 107 p. Dissertação (Mestrado), Faculdade de Ciências Agrárias e Veterinárias, Universidade Estadual Paulista.

5 CHAIM, A. Aperfeiçoamento de um bico de pulverização eletrostática para geração de gotas com alto nível de carga. Pesquisa Agropecuária Brasileira, Brasília, v.33, n.4, p.463 - 470, 1998.

6 ChAIM, A.; BOTTON M.; SCRAMIN, S.; PESSOA, M. C. P. Y.; SANHUEZA, R. M. V; KOVALESKI, A. Deposição de agrotóxicos pulverizados na cultura da maçã. Pesquisa Agropecuária Brasileira, Brasília, v. 38, p. 889-892, 2003.

7 CHAIM, A.; CASTRO, V. L. S. S.; CORRALES, F. M.; GALVÃO, J. A. H.; CABRAL, O. M. R.; NICOLELLA, G. Método para monitorar perdas na aplicação de agrotóxicos na cultura do tomate. Pesquisa Agropecuária Brasileira, Brasília, v.34, n.5, p.741 - 747, 1999b.

8 CHAIM, A.; LARANJEIRO, A. J.; CAPALBO, D. M. F. Bico pneumático eletrostático para aplicação de inseticidas biológicos em floresta de eucalipto. Jaguariúna: Embrapa Meio Ambiente, 1999. 33 p. (Embrapa Meio Ambiente, Boletim de Pesquisa, 3).

9 CHAIM, A.; PESSOA, M. C. P. Y.; FERRACINI, V. L. Eficiência de deposição de agrotóxicos, obtida com bocal eletrostático para pulverizador motorizado costal. Pesquisa Agropecuária Brasileira, Brasília,v.37, n.4, p.497-501, 2002.

10 CHAIM, A.; PESSOA, M. C. P. Y.; FERRACINI, V. L. Eficiência de pulverização em videira, comparando bicos e pulverizadores. Pesticidas: revista de ecotoxicologia e meio ambiente. Curitiba, v.14, p.39 - 46, 2004.

11 CHAIM, A.; VALARINI, P.J.; OLIVEIRA, D.A.; MORSOLETO, R.V.; PIO, L.C. Avaliação de perdas de pulverização em culturas de feijão e tomate. Jaguariúna: Embrapa Meio Ambiente, 1999a. 29 p. (Embrapa Meio Ambiente, Boletim de Pesquisa, 2).

12 CHAIM, A.; VALARINI, P. J.; PIO, L. C. Avaliação de perdas na pulverização de agrotóxicos na cultura do feijão. Pesticidas: revista de ecotoxicologia e meio ambiente, Curitiba, v.10, p.13 - 22, 2000.

13 COFFEE, R.A. Electrodynamic crop spraying. Outlook on Agriculture, Elmsford, v.10, n.7, p.350-356, 1981.

14 PESSOA, M. C. P. Y.; CHAIM, A. Programa computacional para estimativa de uniformidade de gotas de pulverização aérea. Pesquisa Agropecuária Brasileira, Brasília, v.34, n.1, p.45 - 56, 1999.

15 SCRAMIN, S.; ChAIM, A.; PESSOA, M. C. P. Y., FERRACINI, V. L.; ANTÔNIO, P. L.; ALVARENGA, N. Avaliação de bicos de pulverização de agrotóxicos na cultura do algodão. Pesticidas: revista de ecotoxicologia e meio ambiente. Curitiba, v.12, p.43 - 50, 2002. 